MATHEMATICS OF COMPUTATION

Volume 76, Number 257, January 2007, Pages 1-18

S $0025-5718(06) 01889-8$

Article electronically published on August 1, 2006

\title{
NONCONFORMING TETRAHEDRAL FINITE ELEMENTS FOR FOURTH ORDER ELLIPTIC EQUATIONS
}

\author{
WANG MING AND JINCHAO XU
}

\begin{abstract}
This paper is devoted to the construction of nonconforming finite elements for the discretization of fourth order elliptic partial differential operators in three spatial dimensions. The newly constructed elements include two nonconforming tetrahedral finite elements and one quasi-conforming tetrahedral element. These elements are proved to be convergent for a model biharmonic equation in three dimensions. In particular, the quasi-conforming tetrahedron element is a modified Zienkiewicz element, while the nonmodified Zienkiewicz element (a tetrahedral element of Hermite type) is proved to be divergent on a special grid.
\end{abstract}

\section{INTRODUCTION}

The construction of appropriate finite element spaces for fourth order elliptic partial differential equations is an intriguing subject. This problem has been wellstudied in two-dimensional spaces, and there have been a lot of interesting constructions of both conforming and nonconforming finite element spaces. In comparison, there has been very little work devoted to three-dimensional problems.

A conforming finite element space for fourth order problems consist of piecewise polynomials that are globally continuously differentiable $\left(C^{1}\right)$. This smoothness requirement can only be met with piecewise polynomials of sufficiently high degree. In two dimensions, it is known 31 that at least a 5th degree polynomial (the well-known Argyris element) is needed on a triangular mesh. Such a high degree polynomial leads to finite element spaces with a very large degree of freedom which is not computationally desirable. As a result, many lower degree nonconforming finite elements have been constructed and used in practice (see [8]).

In three spatial dimensions, even higher degree polynomials are needed to construct a conforming finite element space on, say, a tetrahedral finite element grid. In [30] (see also [17]), a conforming tetrahedral conforming finite element space was first constructed using the 9th degree of polynomials. This element requires $C^{1}$ globally, $C^{2}$ on all element edges, and $C^{4}$ on all element vertices. The degree of freedom for this element is huge, 220 on each element! In order to reduce the degree

Received by the editor October 8, 2004 and, in revised form, September 16, 2005.

2000 Mathematics Subject Classification. Primary 65N30.

Key words and phrases. Nonconforming finite element, 3-dimension, fourth order elliptic equation, biharmonic.

The work of the first author was supported by the National Natural Science Foundation of China (10571006).

The work of the second author was supported by National Science Foundation DMS-0209479 and DMS-0215392 and the Changjiang Professorship through Peking University. 
of polynomials, as in two dimensions, there has been some work on the construction of conforming finite element spaces on macro-elements (namely by further partitioning a tetrahedron into sub-tetrahedrons); see [1] and 29] (similar to Clough-Tocher in two dimensions) and [14. But these elements all still have a very large degree of freedom, and furthermore the macro-elements are often awkward to use in practical applications.

To reduce the degree of polynomials and degree of freedom on each element, one naturally turns to nonconforming elements. Surprisingly, there has been very little work on the construction of nonconforming finite elements for fourth order elliptic boundary value problems in three dimensions. The purpose of this work is to fill in this important gap in the literature for this type of element.

The construction of nonconforming finite elements for fourth order problems in three dimensions is not only important from a mathematical point of view but also potentially important in practical applications. Indeed, two-dimensional biharmonic equations have been much used in modeling linear plates (see [15]), and such practical applications contributed to the importance and interest of studying efficient numerical methods such as nonconforming finite elements to solve this type of equation. We would like to point out that the three-dimensional biharmonic operator also has important applications in practice. One notable example is the Cahn-Hilliard diffusion equation (see [6]) and its modified version (see [13] and the references there). The complex microstructure evolutions for many important material processes, such as the phase separation in binary alloys and the solidifications of metals and alloys (see [5]), can be modeled by the Cahn-Hilliard diffusion equations.

There were many works on the numerical methods for the Cahn-Hilliard equation; see [2, 3, 5, , 9- 12, [19] and their references. In addition to the finite difference method and also the spectral method, the fourth order term in the CahnHilliard equation can also be discretized by the finite element method (see [2, 3, [10]-12]). The finite element methods of mixed type, namely by writing the biharmonic operator as a product of two Laplacian operators, were discussed in 2, 3, 11. It is conceivable that the biharmonic operator can also be discretized directly from its original form, as it is often done for biharmonic equations in two dimensions. This kind of finite element method had been applied to Cahn-Hilliard equation in one and two dimensions (see [10, 12]), and there is no work for three dimensions yet. As discussed above, the existing 3-dimensional conforming finite elements are not very practical and the nonconforming finite element methods proposed in this paper can hopefully be used for such applications.

In this paper, we will propose some finite elements for three-dimensional fourth order partial differential equations. We took the natural approach of trying to extend the various nonconforming finite element in two dimensions to three dimensions. In two dimensions, there are well-known nonconforming elements, including the elements named after Morley, Zienkiewicz, Adini, Bogner-Fox-Schmit, etc. (see [4, 8, 16, 18]). There are some other ways of constructing elements, such as the quasi-conforming method [25, 7]. In this paper, we will focus on tetrahedral complete or incomplete cubic elements, and propose and analyze the following three types of elements:

(1) A cubic tetrahedral element with 20 degrees of freedom and complete cubic polynomial shape function space. 
(2) A incomplete cubic tetrahedral element with 16 degrees of freedom and incomplete cubic polynomial shape function space.

(3) A quasi-conforming tetrahedral element with 16 degrees of freedom similar to a nine-parameter quasi-conforming element.

The first two are nonconforming elements, and the last one is an element constructed by the quasi-conforming method. For nonconforming elements, the basic mathematical theory has been studied in many works (see [8, 16, 22, 24, 33). For quasi-conforming elements, detailed discussions can be found in [32, 33]. Following these theories, we give the convergence analysis of the elements.

The element of Hermite tetrahedron of type $\left(3^{\prime}\right)$ in $[8$, called a three-dimensional Zienkiewicz element in this paper, is also viewed as an element for biharmonic equations just like the two-dimensional Zienkiewicz element. In two-dimensional case, the Zienkiewicz element is not convergent for general meshes. We will also show that the three-dimensional Zienkiewicz element is divergent for some popular grids in three dimensions.

We note that the degree of freedom of each element proposed in this paper is substantially smaller than any known conforming elements. We expect that they can be easily used in practice.

The rest of the paper is organized as follows. Section 2 gives a basic description of the nonconforming element method. Section 3 gives a detailed description of the new finite elements. Section 4 shows the convergence of the new elements and the divergence of the three-dimensional Zienkiewicz element. Some concluding remarks are made at the end of the paper.

\section{Preliminaries}

In this section, we shall give a brief discussion of a model fourth order elliptic boundary value problem and how it may be discretized by a nonconforming finite element method.

Given a bounded polyhedron domain $\Omega \subset R^{3}$ with boundary $\partial \Omega$, for a nonnegative integer $s$, let $H^{s}(\Omega),\|\cdot\|_{s, \Omega}$, and $|\cdot|_{s, \Omega}$ be the usual Sobolev space, norm, and seminorm, respectively. Let $H_{0}^{s}(\Omega)$ be the closure of $C_{0}^{\infty}(\Omega)$ in $H^{s}(\Omega)$ with respect to the norm $\|\cdot\|_{s, \Omega}$ and $(\cdot, \cdot)$ denote the inner product of $L^{2}(\Omega)$.

For $f \in L^{2}(\Omega)$, we consider the following fourth order boundary value problem:

$$
\left\{\begin{array}{l}
\Delta^{2} u=f, \quad \text { in } \Omega, \\
\left.u\right|_{\partial \Omega}=\left.\frac{\partial u}{\partial \nu}\right|_{\partial \Omega}=0,
\end{array}\right.
$$

where $\nu=\left(\nu_{1}, \nu_{2}, \nu_{3}\right)^{\top}$ is the unit outer normal to $\partial \Omega$ and $\Delta$ is the standard Laplacian operator.

For any function $v \in H^{1}(T)$, set

$$
D v=\left(\frac{\partial v}{\partial x_{1}}, \frac{\partial v}{\partial x_{2}}, \frac{\partial v}{\partial x_{3}}\right) .
$$

When $v \in H^{2}(\Omega)$, we define

$$
E(v)=\left(\frac{\partial^{2} v}{\partial x_{1}^{2}}, \frac{\partial^{2} v}{\partial x_{2}^{2}}, \frac{\partial^{2} v}{\partial x_{3}^{2}}, \frac{\partial^{2} v}{\partial x_{1} \partial x_{2}}, \frac{\partial^{2} v}{\partial x_{1} \partial x_{3}}, \frac{\partial^{2} v}{\partial x_{2} \partial x_{3}}\right)^{\top} .
$$


Let $K \in R^{6 \times 6}$ be the matrix given by

$$
K=\operatorname{diag}(1,1,1,2,2,2) .
$$

Define

$$
a(v, w)=\int_{\Omega} E(w)^{\top} K E(v), \quad \forall v, w \in H^{2}(\Omega) .
$$

The weak form of problem (2.1) is: find $u \in H_{0}^{2}(\Omega)$ such that

$$
a(u, v)=(f, v), \quad \forall v \in H_{0}^{2}(\Omega) .
$$

For a subset $B \subset R^{3}$ and a nonnegative integer $r$, let $P_{r}(B)$ be the space of all polynomials of degree not greater than $r$, and let $Q_{r}(B)$ the space of all polynomials of degree in each coordinate not greater than $r$.

Let $\left(T, P_{T}, \Phi_{T}\right)$ be a finite element where $T$ is the geometric shape, $P_{T}$ the shape function space, and $\Phi_{T}$ the vector of degrees of freedom, and let $\Phi_{T}$ be $P_{T^{-}}$ unisolvent (see [8]). Let $\mathcal{T}_{h}$ be a triangulation of $\Omega$ with mesh size $h$. For each element $T \in \mathcal{T}_{h}$, let $h_{T}$ be the diameter of the smallest ball containing $T$ and let $\rho_{T}$ be the diameter of the largest ball contained in $T$.

Let $\left\{\mathcal{T}_{h}\right\}$ be a family of triangulations with $h \rightarrow 0$. Throughout the paper, we assume that $\left\{\mathcal{I}_{h}\right\}$ is quasi-uniform, namely, it satisfies that $h_{T} \leq h \leq \eta \rho_{T}, \forall T \in \mathcal{T}_{h}$ for a positive constant $\eta$ independent of $h$.

For each $\mathcal{T}_{h}$, let $V_{h 0}$ be the corresponding finite element space associated with $\left(T, P_{T}, \Phi_{T}\right)$ for the discretization of $H_{0}^{2}(\Omega)$. This defines a family of finite element spaces $\left\{V_{h 0}\right\}$. In the case of a nonconforming element, $V_{h 0} \not \subset H_{0}^{2}(\Omega)$.

For $v, w \in H^{2}(\Omega)+V_{h 0}$, we define

$$
a_{h}(v, w)=\sum_{T \in \mathcal{T}_{h}} \int_{T} E(w)^{\top} K E(v) .
$$

The finite element method for problem (2.4) corresponding to element $\left(T, P_{T}, \Phi_{T}\right)$ is: find $u_{h} \in V_{h 0}$ such that

$$
a_{h}\left(u_{h}, v_{h}\right)=\left(f, v_{h}\right), \quad \forall v_{h} \in V_{h 0} .
$$

We introduce the following mesh-dependent norm $\|\cdot\|_{m, h}$ and seminorm $|\cdot|_{m, h}$ :

$$
\|v\|_{m, h}=\left(\sum_{T \in \mathcal{T}_{h}}\|v\|_{m, T}^{2}\right)^{1 / 2}, \quad|v|_{m, h}=\left(\sum_{T \in \mathcal{T}_{h}}|v|_{m, T}^{2}\right)^{1 / 2}
$$

for all functions $v \in L^{2}(\Omega)$ with $v^{T} \in H^{m}(T), \forall T \in \mathcal{T}_{h}$.

For each element $T \in \mathcal{T}_{h}$, let $\Pi_{T}$ denote the canonical interpolation operator of $\left(T, P_{T}, \Phi_{T}\right)$, and define $\Pi_{h}$ by $\left.\left(\Pi_{h} v\right)\right|_{T}=\Pi_{T}\left(\left.v\right|_{T}\right)$, where $T \in \mathcal{T}_{h}$ and $v$ is piecewise smooth.

\section{Tetrahedral elements}

Given a tetrahedron $T$ with vertices $a_{i}=\left(x_{i 1}, x_{i 2}, x_{i 3}\right)^{\top}, 0 \leq i \leq 3$, denote by $F_{i}$ the facet opposite $a_{i}$, by $b_{i}$ the barycenter of $F_{i}, 0 \leq i \leq 3$, and by $\lambda_{0}, \cdots, \lambda_{3}$ the barycentric coordinates of $T$.

Let $\hat{T}$ be the reference tetrahedron with vertices $\hat{a}_{i}$ given by

$$
\hat{a}_{0}=(0,0,0)^{\top}, \hat{a}_{1}=(1,0,0)^{\top}, \hat{a}_{2}=(0,1,0)^{\top}, \hat{a}_{3}=(0,0,1)^{\top} .
$$


Set

$$
B_{T}=\left(\begin{array}{lll}
x_{11}-x_{01} & x_{21}-x_{01} & x_{31}-x_{01} \\
x_{12}-x_{02} & x_{22}-x_{02} & x_{32}-x_{02} \\
x_{13}-x_{03} & x_{23}-x_{03} & x_{33}-x_{03}
\end{array}\right)=\left(a_{1}-a_{0}, a_{2}-a_{0}, a_{3}-a_{0}\right),
$$

and $F_{T} \hat{x}=B_{T} \hat{x}+a_{0}, \hat{x} \in R^{3} ;$ then

$$
T=F_{T} \hat{T}, \quad a_{i}=F_{T} \hat{a}_{i}, \quad 0 \leq i \leq 3 .
$$

Set $B_{T}^{-1}=\left(\xi_{i j}\right)_{3 \times 3}$. Let $B_{1}, B_{2}, B_{3}$ be the row vectors of $B_{T}^{-1}$ and

$$
B_{0}=-\left(B_{1}+B_{2}+B_{3}\right)
$$

then

$$
D \lambda_{i}=B_{i}, \quad 0 \leq i \leq 3
$$

3.1. The cubic tetrahedral element. For the first nonconforming element, called the cubic tetrahedral element, $\left(T, P_{T}, \Phi_{T}\right)$ is defined by (see Figure 1)

1) $T$ is a tetrahedron,

2) $P_{T}=P_{3}(T)$

3) $\Phi_{T}$ is the degree of freedom vector with components

$$
v\left(a_{j}\right), \frac{\partial v}{\partial \nu}\left(b_{j}\right), 0 \leq j \leq 3, D v\left(a_{i}\right)\left(a_{j}-a_{i}\right), 0 \leq i \neq j \leq 3, \forall v \in C^{1}(T) .
$$

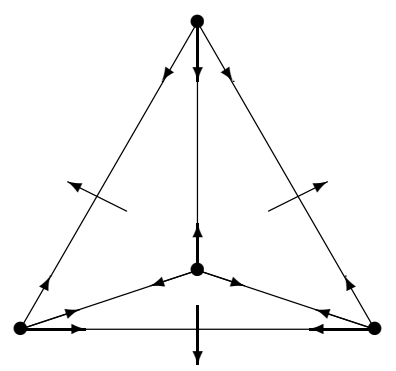

FiguRe 1

We claim that $\Phi_{T}$ is $P_{T}$-unisolvent since, with respect to $\Phi_{T}$, we can obtain the following basis functions of $P_{T}$ :

$$
\begin{cases}q_{i}=\frac{9}{4\left\|B_{i}\right\|}\left(\sum_{\substack{0 \leq j<k<l \leq 3 \\ j \neq i, k \neq i, l \neq i}} \lambda_{j} \lambda_{k} \lambda_{l}-\lambda_{i} \sum_{\substack{0 \leq j<k \leq 3 \\ j \neq i, k \neq i}} \lambda_{j} \lambda_{k}\right), & 0 \leq i \leq 3, \\ p_{i}=3 \lambda_{i}^{2}-2 \lambda_{i}^{3}+\sum_{\substack{0 \leq k \leq 3 \\ k \neq i}} \frac{4 B_{i} B_{k}^{\top}}{3\left\|B_{k}\right\|} q_{k}, & 0 \leq i \leq 3, \\ p_{i j}=\lambda_{i}^{2} \lambda_{j}+\frac{\left\|B_{j}\right\|}{9} q_{j}+\sum_{\substack{0 \leq k \leq 3 \\ k \neq i, k \neq j}} \frac{\left(2 B_{i}+B_{j}\right) B_{k}^{\top}}{9\left\|B_{k}\right\|} q_{k}, & 0 \leq i \neq j \leq 3 .\end{cases}
$$


In fact, by a direct calculation (see below), we have that

$$
\left\{\begin{array}{lll}
q_{i}\left(a_{k}\right)=0, & D q_{i}\left(a_{k}\right)=0, & \\
p_{i}\left(a_{k}\right)=\delta_{i k}, & D p_{i}\left(a_{k}\right)=0, & \frac{\partial p_{i}}{\partial \nu}\left(b_{k}\right)=\delta_{i k}, \\
p_{i j}\left(a_{k}\right)=0, & D p_{i j}\left(a_{k}\right)\left(a_{l}-a_{k}\right)=\delta_{i k} \delta_{j l}, & \frac{\partial p_{i j}}{\partial \nu}\left(b_{k}\right)=0,
\end{array}\right.
$$

when $0 \leq i \neq j \leq 3$ and $0 \leq k \neq l \leq 3$, and where $\delta_{i k}$ and $\delta_{j l}$ are Kronecker deltas.

The corresponding interpolation operator $\Pi_{T}$ can be written by, $\forall v \in C^{1}(T)$

$$
\Pi_{T} v=\sum_{0 \leq i \leq 3} p_{i} v\left(a_{i}\right)+\sum_{0 \leq i \leq 3} q_{i} \frac{\partial v}{\partial \nu}\left(b_{i}\right)+\sum_{0 \leq i \neq j \leq 3} p_{i j} D v\left(a_{i}\right)\left(a_{j}-a_{i}\right) .
$$

Now we verify (3.3). For the function $q_{0}$, we have

$$
\begin{aligned}
& q_{0}=\frac{9}{4\left\|B_{0}\right\|}\left(\lambda_{1} \lambda_{2} \lambda_{3}-\lambda_{0}\left(\lambda_{1} \lambda_{2}+\lambda_{2} \lambda_{3}+\lambda_{3} \lambda_{1}\right)\right), \\
& D q_{0}=\frac{9}{4\left\|B_{0}\right\|}\left(\lambda_{2} \lambda_{3} D \lambda_{1}+\lambda_{1} \lambda_{3} D \lambda_{2}+\lambda_{1} \lambda_{2} D \lambda_{3}\right. \\
& \left.-\left(\lambda_{1} \lambda_{2}+\lambda_{2} \lambda_{3}+\lambda_{3} \lambda_{1}\right) D \lambda_{0}-\lambda_{0} \sum_{1 \leq i \neq j \leq 3} \lambda_{i} D \lambda_{j}\right) .
\end{aligned}
$$

Since $\lambda_{i}\left(a_{j}\right)=\delta_{i j}$ we obviously have $q_{0}\left(a_{j}\right)=0$ and $D q_{0}\left(a_{j}\right)=0(0 \leq j \leq 3)$. Furthermore,

$$
\begin{aligned}
& D q_{0}\left(b_{0}\right)=\frac{1}{4\left\|B_{0}\right\|}\left(D \lambda_{1}+D \lambda_{2}+D \lambda_{3}-3 D \lambda_{0}\right)=-\frac{D \lambda_{0}}{\left\|B_{0}\right\|}=-\frac{B_{0}}{\left\|B_{0}\right\|}, \\
& D q_{0}\left(b_{j}\right)=\frac{1}{4\left\|B_{0}\right\|}\left(D \lambda_{j}-D \lambda_{0}-2 D \lambda_{j}-\sum_{\substack{1 \leq i \leq 3 \\
i \neq j}} D \lambda_{i}\right)=0,1 \leq j \leq 3 .
\end{aligned}
$$

Thanks to (3.1) the outer normal of each $F_{i}$ is just $-B_{i}$. Hence $\frac{\partial}{\partial \nu} q_{0}\left(b_{j}\right)=\delta_{0 j}$, $0 \leq j \leq 3$. For other $q_{i}$ we can use a completely similar argument. Thus the first line of (3.3) is verified.

Let $0 \leq i, k \leq 3$. Obviously $p_{i}\left(a_{k}\right)=\delta_{i k}$ and

$$
D p_{i}=6\left(1-\lambda_{i}\right) \lambda_{i} D \lambda_{i}+\sum_{\substack{0 \leq j \leq 3 \\ j \neq i}} \frac{4 B_{i} B_{j}^{\top}}{3\left\|B_{j}\right\|} D q_{j}
$$

This directly leads to the fact that $D p_{i}\left(a_{k}\right)=0$. By the properties of $q_{j}$, we have $D p_{i}\left(b_{i}\right)=0$ and

$$
D p_{i}\left(b_{k}\right) B_{k}^{\top}=\frac{4}{3} B_{i} B_{k}^{\top}+\frac{4 B_{i} B_{k}^{\top}}{3\left\|B_{k}\right\|} D q_{k}\left(b_{k}\right) B_{k}^{\top}=0
$$

when $k \neq i$. Thus the second line of (3.3) is also verified.

Finally, we consider $p_{i j}$. Let $0 \leq i \neq j \leq 3$ and $0 \leq k \leq 3$. Then $p_{i j}\left(a_{k}\right)=0$ by definition, and

$$
D p_{i j}=2 \lambda_{i} \lambda_{j} D \lambda_{i}+\lambda_{i}^{2} D \lambda_{j}+\frac{\left\|B_{j}\right\|}{9} D q_{j}+\sum_{\substack{0 \leq m \leq 3 \\ m \neq i, m \neq j}} \frac{\left(2 B_{i}+B_{j}\right) B_{m}^{\top}}{9\left\|B_{m}\right\|} D q_{m} .
$$


Now, for $l \neq k$, since $D \lambda_{j}\left(a_{l}-a_{k}\right)=\delta_{j l}-\lambda_{j}\left(a_{k}\right)$ (see [8, p. 65]), we have

$$
D p_{i j}\left(a_{k}\right)\left(a_{l}-a_{k}\right)=\lambda_{i}^{2}\left(a_{k}\right) D \lambda_{j}\left(a_{l}-a_{k}\right)=\delta_{i k}\left(\delta_{j l}-\delta_{j k}\right)=\delta_{i k} \delta_{j l} .
$$

Using the properties of $q_{m}, 0 \leq m \leq 3$, we deduce that

$$
\begin{aligned}
& D p_{i j}\left(b_{i}\right) B_{i}^{\top}=0, \quad D p_{i j}\left(b_{j}\right) B_{j}^{\top}=\frac{\left\|B_{j}\right\|}{9}\left(\left\|B_{j}\right\|+D q_{j}\left(b_{j}\right) B_{j}^{\top}\right)=0, \\
& D p_{i j}\left(b_{k}\right) B_{k}^{\top}=\frac{2}{9} B_{i} B_{k}^{\top}+\frac{1}{9} B_{j} B_{k}^{\top}+\frac{\left(2 B_{i}+B_{j}\right) B_{k}^{\top}}{9\left\|B_{k}\right\|} D q_{k}\left(b_{k}\right) B_{k}^{\top}=0, k \neq i, j .
\end{aligned}
$$

Hence the last line of (3.3) is verified.

For $0 \leq i \leq 3$, vectors $a_{j}-a_{i}, j \neq i$, form a basis of $R^{3}$. Thus the degrees of freedom of the cubic tetrahedral element can be replaced by

$$
v\left(a_{j}\right), D v\left(a_{j}\right), \frac{\partial v}{\partial \nu}\left(b_{j}\right), 0 \leq j \leq 3, \forall v \in C^{1}(T) .
$$

For the cubic tetrahedral element, we can define the corresponding finite element space $V_{h 0}$ as follows: $v \in V_{h 0}$ if any only if (1) $\left.v\right|_{T} \in P_{3}(T), \forall T \in T_{h},(2) v$ and $D v$ are continuous at all vertices of elements in $\mathcal{T}_{h}$ and vanish at all vertices belonging to $\partial \Omega$, and (3) $\frac{\partial}{\partial \nu} v$ is continuous at the barycenters of all facets of elements in $\mathcal{T}_{h}$ and vanishes at barycenters of all facets on $\partial \Omega$.

Unlike the Zienkiewicz element, this complete cubic finite element space is not always contained in $C^{0}(\bar{\Omega})$. To see this, let us choose two different elements $T, T^{\prime} \in$ $\mathcal{T}_{h}$ such that they have a common facet $F$ and $T \cup T^{\prime} \subset \Omega$. Denote by $b$ the barycenter of $F$ and by $\nu$ the unit outer normal to $F$ with respective to $T$. Let $v_{h}$ be the function in $V_{h 0}$ satisfying: $v_{h} \equiv 0$ outside $T \cup T^{\prime}$ and $\frac{\partial}{\partial \nu} v_{h}(b)=1$. Then $\left.v_{h}\right|_{T}$ is just one of the basis functions $q_{i}$ given by (3.2). From (3.2) $\left.v_{h}\right|_{T}$ does not vanish at all relative inner points of each facet of $T$. Hence $v_{h}$ is not continuous through the facets of $T$ different from $F$.

Lemma 3.1. Let $V_{h 0}$ be the finite element space of the cubic tetrahedral element. If $T, T^{\prime} \in \mathcal{T}_{h}$ have a common facet $F$, then

$$
\int_{F} D\left(\left.v_{h}\right|_{T}\right)=\int_{F} D\left(\left.v_{h}\right|_{T^{\prime}}\right), \quad v_{h} \in V_{h 0} .
$$

If a facet $F$ of $T \in \mathcal{T}_{h}$ is on $\partial \Omega$, then

$$
\int_{F} D\left(\left.v_{h}\right|_{T}\right)=0, \quad v_{h} \in V_{h 0} .
$$

Proof. Let $v_{h} \in V_{h 0}$ and let $F$ be the common facet of $T, T^{\prime} \in \mathcal{T}_{h}$. Denote the unit normal of $F$ relative to $T$ by $\nu$, and choose $\nu, \tau^{(1)}, \tau^{(2)}$ as an orthogonal unit basis of $R^{3}$. Let $\tilde{a}_{1}, \tilde{a}_{2}, \tilde{a}_{3}$ be vertices of $F$ and let $\tilde{b}_{0}$ be barycenter of $F$. Denote by $\tilde{\lambda}_{i}$ the area coordinate of $F$ corresponding to vertex $\tilde{a}_{i}$. Then

$$
\begin{aligned}
& \int_{F} \tilde{\lambda}_{j}^{2}=\frac{|F|}{6}=\frac{|F|}{12}\left(\sum_{i=1}^{3} \tilde{\lambda}_{j}^{2}\left(\tilde{a}_{i}\right)+9 \tilde{\lambda}_{j}^{2}\left(\tilde{b}_{0}\right)\right), 1 \leq j \leq 3, \\
& \int_{F} \tilde{\lambda}_{j} \tilde{\lambda}_{k}=\frac{|F|}{12}=\frac{|F|}{12}\left(\sum_{i=1}^{3} \tilde{\lambda}_{j} \tilde{\lambda}_{k}\left(\tilde{a}_{i}\right)+9 \tilde{\lambda}_{j} \tilde{\lambda}_{k}\left(\tilde{b}_{0}\right)\right), 1 \leq j \neq k \leq 3 .
\end{aligned}
$$


From the fact that $P_{2}(F)=\operatorname{span}\left\{\tilde{\lambda}_{1}^{2}, \tilde{\lambda}_{2}^{2}, \tilde{\lambda}_{3}^{2}, \tilde{\lambda}_{1} \tilde{\lambda}_{2}, \tilde{\lambda}_{2} \tilde{\lambda}_{3}, \tilde{\lambda}_{3} \tilde{\lambda}_{1}\right\}$, we obtain

$$
\int_{F} p=\frac{|F|}{12}\left(\sum_{i=1}^{3} p\left(\tilde{a}_{i}\right)+9 p\left(\tilde{b}_{0}\right)\right), \quad \forall p \in P_{2}(F) .
$$

By the definition of $V_{h 0}, \frac{\partial}{\partial \nu}\left(\left.v_{h}\right|_{T}\right)$ and $\frac{\partial}{\partial \nu}\left(\left.v_{h}\right|_{T^{\prime}}\right)$ are quadratic polynomials on $F$, and they equal $\frac{\partial}{\partial \nu} v_{h}$ at $\tilde{b}_{0}$ and $\tilde{a}_{i}, 1 \leq i \leq 3$. Hence

$$
\int_{F} \frac{\partial\left(\left.v_{h}\right|_{T}\right)}{\partial \nu}=\frac{|F|}{12}\left(\sum_{i=1}^{3} \frac{\partial v_{h}}{\partial \nu}\left(\tilde{a}_{i}\right)+9 \frac{\partial v_{h}}{\partial \nu}\left(\tilde{b}_{0}\right)\right)=\int_{F} \frac{\partial\left(\left.v_{h}\right|_{T^{\prime}}\right)}{\partial \nu} .
$$

Denote all sides of $F$ by $S_{1}, S_{2}, S_{3}$, and the unit out normal of $S_{i}$ by $n^{(i)}$, viewed as the boundary of a triangle in the two-dimensional space spanned by directions $\tau^{(1)}$ and $\tau^{(2)}$. Then for $i \in\{1,2\}$ Green's formula gives

$$
\int_{F} \frac{\partial\left(\left.v_{h}\right|_{T}\right)}{\partial \tau^{(i)}}=\left.\sum_{j=1}^{3} n_{i}^{(j)} \int_{S_{j}} v_{h}\right|_{T}, \quad \int_{F} \frac{\partial\left(\left.v_{h}\right|_{T^{\prime}}\right)}{\partial \tau^{(i)}}=\left.\sum_{j=1}^{3} n_{i}^{(j)} \int_{S_{j}} v_{h}\right|_{T^{\prime}} .
$$

By the definition of $V_{h 0},\left.v_{h}\right|_{T}=\left.v_{h}\right|_{T^{\prime}}$ on $S_{j}$. Therefore

$$
\int_{F} \frac{\partial\left(\left.v_{h}\right|_{T}\right)}{\partial \tau^{(i)}}=\int_{F} \frac{\partial\left(\left.v_{h}\right|_{T^{\prime}}\right)}{\partial \tau^{(i)}}, \quad i=1,2 .
$$

Equality (3.5) follows from (3.8) and (3.9). Similarly, we can show (3.6).

3.2. The incomplete cubic tetrahedral element. We shall construct a new element by removing the degrees of freedom $\frac{\partial v}{\partial \nu}\left(b_{j}\right)$ from the cubic tetrahedral element.

For $0 \leq i<j<k \leq 3$, let $a_{i j k}=\left(a_{i}+a_{j}+a_{k}\right) / 3$ and let $\nu_{i j k}$ be the unit out normal of the facet with $a_{i}, a_{j}, a_{k}$ as vertices. For $v \in C^{1}(T)$, define $\tilde{\psi}_{i j k}(v) \in R$ by

$$
\tilde{\psi}_{i j k}(v)=\frac{\partial v}{\partial \nu_{i j k}}\left(a_{i j k}\right)-\frac{1}{3} \sum_{l=i, j, k} \frac{\partial v}{\partial \nu_{i j k}}\left(a_{l}\right)
$$

Define

$$
P_{3}^{\prime \prime}(T)=\left\{p \in P_{3}(T) \mid \tilde{\psi}_{i j k}(p)=0,0 \leq i<j<k \leq 3\right\} .
$$

For a linear polynomial $q$, we have

$$
q\left(a_{i j k}\right)-\frac{1}{3} \sum_{l=i, j, k} q\left(a_{l}\right)=0 .
$$

Then $\tilde{\psi}_{i j k}(p)=0$ when $p \in P_{2}(T)$, that is, $P_{2}(T) \subset P_{3}^{\prime \prime}(T)$. For incomplete cubic tetrahedral element, $\left(T, P_{T}, \Phi_{T}\right)$ is defined by (see Figure 2 )

1) $T$ is a tetrahedron,

2) $P_{T}=P_{3}^{\prime \prime}(T)$,

3) $\Phi_{T}$ is the vector with its component the following degrees of freedom,

$$
v\left(a_{j}\right), 0 \leq j \leq 3, \quad D v\left(a_{i}\right)\left(a_{j}-a_{i}\right), 0 \leq i \neq j \leq 3, \forall v \in C^{1}(T) .
$$




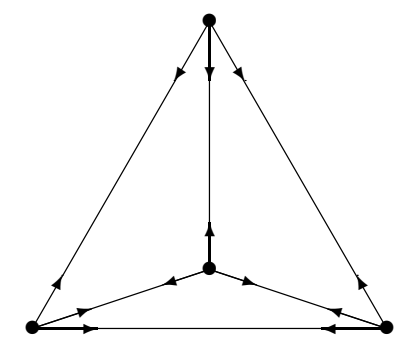

Figure 2

The basis functions of the incomplete cubic tetrahedral element can be derived from ones of the cubic tetrahedral element. Set

$$
\tilde{p}_{i j}=\lambda_{i}^{2} \lambda_{j}-\frac{2\left\|B_{j}\right\|}{9} q_{j}+\sum_{\substack{1 \leq k \leq 4 \\ k \neq i, k \neq j}} \frac{2\left(B_{i}-B_{j}\right) B_{k}^{\top}}{9\left\|B_{k}\right\|} q_{k}, \quad 0 \leq i \neq j \leq 3,
$$

where $\left\{q_{i}\right\}$ are given in (3.2). We can verify that these $\left\{\tilde{p}_{i j}\right\}$, together with the $\left\{p_{i}\right\}$ given in (3.2), form a basis for the incomplete cubic tetrahedral element. The corresponding interpolation operator $\Pi_{T}$ can be written by

$$
\Pi_{T} v=\sum_{0 \leq i \leq 3} p_{i} v\left(a_{i}\right)+\sum_{0 \leq i \neq j \leq 3} \tilde{p}_{i j} D v\left(a_{i}\right)\left(a_{j}-a_{i}\right), \quad \forall v \in C^{1}(T) .
$$

For the incomplete cubic tetrahedral element, we can define the corresponding finite element space $V_{h 0}$ as follows: $V_{h 0}=\left\{v \in L^{2}(\Omega)|v|_{T} \in P_{3}^{\prime \prime}(T), \forall T \in T_{h}, v\right.$ and $D v$ are continuous at all vertices of elements in $\mathcal{T}_{h}$ and vanish at all vertices belonging to $\partial \Omega\}$.

Similar to the cubic tetrahedral element, $V_{h 0}$ here is still not a subspace of $C^{0}(\bar{\Omega})$.

3.3. The three-dimensional Zienkiewicz element. The incomplete tetrahedral element above is reminiscent to the three-dimensional Zienkiewicz element, the element of Hermite tetrahedron of type $\left(3^{\prime}\right)$ in [8]. We shall now discuss this element and its relevant questions.

For $0 \leq i<j<k \leq 3$, define

$$
\psi_{i j k}(v)=6 v\left(a_{i j k}\right)-2 \sum_{l=i, j, k} v\left(a_{l}\right)+\sum_{l=i, j, k} D v\left(a_{l}\right)\left(a_{l}-a_{i j k}\right) .
$$

Define

$$
P_{3}^{\prime}(T)=\left\{p \in P_{3}(T) \mid \psi_{i j k}(p)=0,0 \leq i<j<k \leq 3\right\} .
$$

For the three-dimensional Zienkiewicz element, $\left(T, P_{T}, \Phi_{T}\right)$ is given as follows:

1) The element $T$ is a tetrahedron.

2) The shape function space $P_{T}=P_{3}^{\prime}(T)$.

3) For $v \in C^{1}(T)$, its degree of freedom vector $\Phi_{T}(v)$ is given by

$$
\Phi_{T}(v)=\left(v\left(a_{0}\right), D v\left(a_{0}\right), v\left(a_{1}\right), D v\left(a_{1}\right), v\left(a_{2}\right), D v\left(a_{2}\right), v\left(a_{3}\right), D v\left(a_{3}\right)\right)^{\top} .
$$


The corresponding interpolation operator $\Pi_{T}$ is defined by

$$
\begin{aligned}
\Pi_{T} v= & \sum_{i=0}^{3}\left(3 \lambda_{i}^{2}-2 \lambda_{i}^{3}+2 \lambda_{i} \sum_{\substack{0 \leq j<k \leq 3 \\
j, k \neq i}} \lambda_{j} \lambda_{k}\right) v\left(a_{i}\right) \\
& +\frac{1}{2} \sum_{0 \leq i \neq j \leq 3} \lambda_{i} \lambda_{j}\left(1+\lambda_{i}-\lambda_{j}\right) D v\left(a_{i}\right)\left(a_{j}-a_{i}\right), \quad \forall v \in C^{1}(T) .
\end{aligned}
$$

For the three-dimensional Zienkiewicz element, we can define the corresponding finite element space $V_{h 0}$ as follows: $V_{h 0}=\left\{v \in L^{2}(\Omega)|v|_{T} \in P_{3}^{\prime}(T), \forall T \in T_{h}, v\right.$ and $D v$ are continuous at all vertices of elements in $\mathcal{T}_{h}$ and vanish at all vertices belonging to $\partial \Omega\}$. From [8], we know that $V_{h 0} \subset H^{1}(\Omega)$.

3.4. The quasi-conforming tetrahedral element. The Zienkiewicz element is not convergent in general. We will show in next section that the three-dimensional Zienkiewicz element is also divergent for a special tetrahedral grid. In the twodimensional case, a convergent element was proposed by the so-called quasi-conforming element technique in [25, 7]. Now we use the technique to give a new element by modifying the three-dimensional Zienkiewicz element.

Let $\left(T, P_{T}, \Phi_{T}\right)$ be the three-dimensional Zienkiewicz element. Given a tetrahedron $T$, and let $\Pi_{T}^{1}$ be the linear interpolation operator with the function values at four vertices as degrees of freedom. We define

$$
N_{i j}=\operatorname{span}\left\{1, \frac{1}{2}\left(\lambda_{i}+\lambda_{j}\right)\right\}, \quad 1 \leq i, j \leq 3
$$

For $p \in P_{T}$, define $\partial_{T}^{i j} p \in N^{i j}(1 \leq i, j \leq 3)$ such that for any $q \in N_{i j}$,

$$
\int_{T} q \partial_{T}^{i j} p=\frac{1}{2} \int_{\partial T} q\left(\Pi_{T}^{1} \frac{\partial p}{\partial x_{i}} \nu_{j}+\Pi_{T}^{1} \frac{\partial p}{\partial x_{j}} \nu_{i}\right)-\frac{1}{2} \int_{T}\left(\frac{\partial q}{\partial x_{i}} \frac{\partial p}{\partial x_{j}}+\frac{\partial q}{\partial x_{j}} \frac{\partial p}{\partial x_{i}}\right) .
$$

Set

$$
E_{T}(p)=\left(\partial_{T}^{11} p, \partial_{T}^{22} p, \partial_{T}^{33} p, \partial_{T}^{12} p, \partial_{T}^{13} p, \partial_{T}^{23} p\right)^{\top}
$$

For the quasi-conforming tetrahedral element, we use $E_{T}(p)$ to approximate $E(p)$. Define

$$
N=\left(\begin{array}{cccccc}
\tilde{N}_{11} & & & & & \\
& \tilde{N}_{22} & & & 0 & \\
& & \tilde{N}_{33} & & & \\
& & & \tilde{N}_{12} & & \\
& & & & \tilde{N}_{13} & \tilde{N}_{23}
\end{array}\right)
$$


where $\tilde{N}_{i j}=\left(1, \frac{1}{2}\left(\lambda_{i}+\lambda_{j}\right)\right), 1 \leq i, j \leq 3$,

$$
\begin{aligned}
& H_{T}=\left(\begin{array}{rrrrrr}
\xi_{11}^{2} & \xi_{21}^{2} & \xi_{31}^{2} & 2 \xi_{11} \xi_{21} & 2 \xi_{11} \xi_{31} & 2 \xi_{21} \xi_{31} \\
\xi_{12}^{2} & \xi_{22}^{2} & \xi_{32}^{2} & 2 \xi_{12} \xi_{22} & 2 \xi_{12} \xi_{32} & 2 \xi_{22} \xi_{32} \\
\xi_{13}^{2} & \xi_{23}^{2} & \xi_{33}^{2} & 2 \xi_{13} \xi_{23} & 2 \xi_{13} \xi_{33} & 2 \xi_{23} \xi_{33} \\
\xi_{11} \xi_{12} & \xi_{21} \xi_{22} & \xi_{31} \xi_{32} & \xi_{12} \xi_{21}+\xi_{11} \xi_{22} & \xi_{12} \xi_{31}+\xi_{11} \xi_{32} & \xi_{22} \xi_{31}+\xi_{21} \xi_{32} \\
\xi_{11} \xi_{13} & \xi_{21} \xi_{23} & \xi_{31} \xi_{33} & \xi_{13} \xi_{21}+\xi_{11} \xi_{23} & \xi_{13} \xi_{31}+\xi_{11} \xi_{33} & \xi_{23} \xi_{31}+\xi_{21} \xi_{33} \\
\xi_{12} \xi_{13} & \xi_{22} \xi_{23} & \xi_{32} \xi_{33} & \xi_{13} \xi_{22}+\xi_{12} \xi_{23} & \xi_{13} \xi_{32}+\xi_{12} \xi_{33} & \xi_{23} \xi_{32}+\xi_{22} \xi_{33}
\end{array}\right), \\
& Q=\left(\begin{array}{rrrrrrrrrrrrrrrr}
0 & -32 & 0 & 0 & 0 & 32 & 0 & 0 & 0 & 0 & 0 & 0 & 0 & 0 & 0 & 0 \\
32 & 0 & 4 & 4 & -32 & 24 & -4 & -4 & 0 & 4 & 0 & 0 & 0 & 4 & 0 & 0 \\
0 & 0 & -32 & 0 & 0 & 0 & 0 & 0 & 0 & 0 & 32 & 0 & 0 & 0 & 0 & 0 \\
32 & 4 & 0 & 4 & 0 & 0 & 4 & 0 & -32 & -4 & 24 & -4 & 0 & 0 & 4 & 0 \\
0 & 0 & 0 & -32 & 0 & 0 & 0 & 0 & 0 & 0 & 0 & 0 & 0 & 0 & 0 & 32 \\
32 & 4 & 4 & 0 & 0 & 0 & 0 & 4 & 0 & 0 & 0 & 4 & -32 & -4 & -4 & 24 \\
0 & -16 & -16 & 0 & 0 & 0 & 16 & 0 & 0 & 16 & 0 & 0 & 0 & 0 & 0 & 0 \\
16 & -1 & -1 & 2 & -8 & 4 & 4 & -1 & -8 & 4 & 4 & -1 & 0 & 1 & 1 & 0 \\
0 & -16 & 0 & -16 & 0 & 0 & 0 & 16 & 0 & 0 & 0 & 0 & 0 & 16 & 0 & 0 \\
16 & -1 & 2 & -1 & -8 & 4 & -1 & 4 & 0 & 1 & 0 & 1 & -8 & 4 & -1 & 4 \\
0 & 0 & -16 & -16 & 0 & 0 & 0 & 0 & 0 & 0 & 0 & 16 & 0 & 0 & 16 & 0 \\
16 & 2 & -1 & -1 & 0 & 0 & 1 & 1 & -8 & -1 & 4 & 4 & -8 & -1 & 4 & 4
\end{array}\right),
\end{aligned}
$$

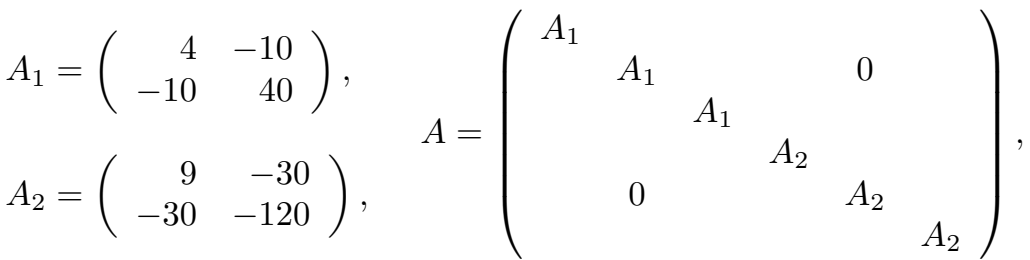

and

$$
M_{T}=\left(\begin{array}{ccccccccc}
1 & 0 & & & & & & \\
0 & B_{T}^{\top} & & & & 0 & & \\
& & 1 & 0 & & & & \\
& & 0 & B_{T}^{\top} & & & & \\
& & & & 1 & 0 & & \\
& & & & 0 & B_{T}^{\top} & & \\
& & 0 & & & & 1 & 0 \\
& & & & & & 0 & B_{T}^{\top}
\end{array}\right) ;
$$

then

$$
E_{T}(p)=\frac{1}{48} H_{T} N A Q M_{T} \Phi_{T}(p), \quad \forall p \in P_{T} .
$$

Now let $V_{h 0}$ be the finite element space corresponding to the three-dimensional Zienkiewicz element. Define

$$
\bar{a}_{h}\left(v_{h}, w_{h}\right)=\sum_{T \in \mathcal{T}_{h}} \int_{T} E_{T}\left(w_{h}\right)^{\top} K E_{T}\left(v_{h}\right), \quad \forall v_{h}, w_{h} \in V_{h 0} .
$$

Instead of solving problem (2.6), the new element finds $\bar{u}_{h} \in V_{h 0}$ such that

$$
\bar{a}_{h}\left(\bar{u}_{h}, v_{h}\right)=\left(f, v_{h}\right), \quad \forall v_{h} \in V_{h 0} .
$$

The quasi-conforming tetrahedral element is a three-dimensional analogue of the element proposed in [25, 7], (see also [33). 
For $v_{h} \in V_{h 0}$ and $i, j \in\{1,2,3\}$, define $\partial_{h}^{i j} v_{h}$ by

$$
\left.\partial_{h}^{i j} v_{h}\right|_{T}=\partial_{T}^{i j}\left(\left.v_{h}\right|_{T}\right), \quad \forall T \in \mathcal{T}_{h} .
$$

Let $\Pi_{T}$ be the interpolation operator of the three-dimensional Zienkiewicz element.

Lemma 3.2. The quasi-conforming tetrahedral element has the following properties:

(1) $E_{T}(p)=E(p), \forall p \in P_{2}(T)$.

(2) There exist positive constants $c_{1}$ and $c_{2}$ independent of $h$ such that

$$
c_{1}|p|_{2, T} \leq \sum_{1 \leq i, j \leq 3}\left|\partial_{T}^{i j} p\right|_{0, T} \leq c_{2}|p|_{2, T}, \quad \forall p \in P_{T}
$$

(3) There exists a constant $C$ independent of $h$ such that

$$
\sum_{1 \leq i, j \leq 3}\left|\frac{\partial^{2} v}{\partial x_{i} \partial x_{j}}-\partial_{T}^{i j} \Pi_{T} v\right|_{0, T} \leq C h|v|_{3, T}, \quad \forall v \in H^{3}(T) .
$$

Proof. For $1 \leq i, j \leq 3$, any $p \in P_{T}$ and any $q \in N_{i j}$, Green's formula gives

$$
\int_{T} q \frac{\partial^{2} p}{\partial x_{i} \partial x_{j}}=\frac{1}{2} \int_{\partial T} q\left(\frac{\partial p}{\partial x_{i}} \nu_{j}+\frac{\partial p}{\partial x_{j}} \nu_{i}\right)-\frac{1}{2} \int_{T}\left(\frac{\partial q}{\partial x_{i}} \frac{\partial p}{\partial x_{j}}+\frac{\partial q}{\partial x_{j}} \frac{\partial p}{\partial x_{i}}\right) .
$$

If $p \in P_{2}(T)$, then $E(p)$ is uniquely determined by (3.20). On the other hand,

$$
\Pi_{T}^{1} \frac{\partial p}{\partial x_{i}}=\frac{\partial p}{\partial x_{i}}, \quad \forall p \in P_{2}(T) .
$$

By (3.13) we obtain that $E_{T}(p)=E(p), \forall p \in P_{2}(T)$.

It can be verified that the rank of matrix $Q$ is 12 . Thus the rank of $A Q M_{T}$ is 12 , too. Let $S$ be the subspace of $R^{16}$ such that $A Q M_{T} d=0, \forall d \in S$. Then the dimension of $S$ is 4 . By conclusion (1) of the lemma, we have

$$
S=\operatorname{span}\left\{\Phi_{T}(1), \Phi_{T}\left(x_{1}\right), \Phi_{T}\left(x_{2}\right), \Phi_{T}\left(x_{3}\right)\right\} .
$$

If $E_{T}(p)=0$ for some $p \in P_{T}$, then $A Q M_{T} \Phi_{T}(p)=0$. It follows that $p \in P_{1}(T)$. Therefore, for all $T \in \mathcal{T}_{h}$,

$$
\alpha_{1 T}|p|_{2, T} \leq \sum_{1 \leq i, j \leq 3}\left|\partial_{T}^{i j} p\right|_{0, T} \leq \alpha_{2 T}|p|_{2, T}, \quad \forall p \in P_{T},
$$

where $\alpha_{1 T}$ and $\alpha_{2 T}$ are positive constants perhaps dependent on $T$. Now define

$$
g(T)=\sup _{\substack{p \in P_{T} \\|p|_{2, T} \neq 0}} \frac{1}{|p|_{2, T}} \sum_{1 \leq i, j \leq 3}\left|\partial_{T}^{i j} p\right|_{0, T} .
$$

By (3.15), (3.1) and the quality

$$
\begin{aligned}
p= & \sum_{i=0}^{3}\left(3 \lambda_{i}^{2}-2 \lambda_{i}^{3}+2 \lambda_{i} \sum_{\substack{0 \leq j<k \leq 3 \\
j, k \neq i}} \lambda_{j} \lambda_{k}\right) p\left(a_{i}\right) \\
& +\frac{1}{2} \sum_{0 \leq i \neq j \leq 3} \lambda_{i} \lambda_{j}\left(1+\lambda_{i}-\lambda_{j}\right) D p\left(a_{i}\right)\left(a_{j}-a_{i}\right), \quad \forall p \in P_{T},
\end{aligned}
$$

we can treat $\left|\partial_{T}^{i j} p\right|_{0, T}$ and $|p|_{2, T}$ as continuous functions with respect to $B_{T}$ and $\Phi_{T}(p)$. Thus function $g(T)$ is a continuous function with respect to matrix $B_{T}$, say 
$g\left(B_{T}\right)$. By (3.21) and the property of continuous function, there exist two positive constants $c_{1}$ and $c_{2}$ such that

$$
\max _{\left\|B_{T}\right\|=1} g\left(B_{T}\right) \leq c_{2}, \quad \min _{\left\|B_{T}\right\|=1} g\left(B_{T}\right) \geq c_{1},
$$

where $\left\|B_{T}\right\|$ is a norm of matrix $B_{T}$.

For $T \in \mathcal{T}_{h}$, define $\tilde{T}=\left\{\tilde{x} \mid \tilde{x}=\left\|B_{T}\right\|^{-1} x, \forall x \in T\right\}$. Then $\left\|B_{\tilde{T}}\right\|=1$ and

$$
c_{1}|\tilde{p}|_{2, \tilde{T}} \leq \sum_{1 \leq i, j \leq 3}\left|\partial_{\tilde{T}}^{i j} \tilde{p}\right|_{0, \tilde{T}} \leq c_{2}|\tilde{p}|_{2, \tilde{T}}, \quad \forall \tilde{p} \in P_{\tilde{T}} .
$$

Given $p \in P_{T}$, let $\tilde{p}(\tilde{x})=p\left(\left\|B_{T}\right\| \tilde{x}\right), \forall \tilde{x} \in \tilde{T}$. Then

$$
|\tilde{p}|_{2, \tilde{T}}=\left\|B_{T}\right\|^{1 / 2}|p|_{2, T} .
$$

On the other hand, by (3.13) we have, $\forall \tilde{q} \in N_{i j}, 1 \leq i, j \leq 3$,

$$
\begin{aligned}
\int_{\tilde{T}} \tilde{q} \partial_{\tilde{T}}^{i j} \tilde{p}= & \frac{1}{2} \int_{\partial \tilde{T}} \tilde{q}\left(\Pi_{\tilde{T}}^{1} \frac{\partial \tilde{p}}{\partial \tilde{x}_{i}} \nu_{j}+\Pi_{\tilde{T}}^{1} \frac{\partial \tilde{p}}{\partial \tilde{x}_{j}} \nu_{i}\right)-\frac{1}{2} \int_{\tilde{T}}\left(\frac{\partial \tilde{q}}{\partial \tilde{x}_{i}} \frac{\partial \tilde{p}}{\partial \tilde{x}_{j}}+\frac{\partial \tilde{q}}{\partial \tilde{x}_{j}} \frac{\partial \tilde{p}}{\partial \tilde{x}_{i}}\right) \\
= & \frac{1}{2\left\|B_{T}\right\|} \int_{\partial T} \tilde{q}\left(\Pi_{T}^{1} \frac{\partial p}{\partial x_{i}} \nu_{j}+\Pi_{T}^{1} \frac{\partial p}{\partial x_{j}} \nu_{i}\right) \\
& \quad-\frac{1}{2\left\|B_{T}\right\|} \int_{T}\left(\frac{\partial \tilde{q}}{\partial x_{i}} \frac{\partial p}{\partial x_{j}}+\frac{\partial \tilde{q}}{\partial x_{j}} \frac{\partial p}{\partial x_{i}}\right)=\frac{1}{\left\|B_{T}\right\|} \int_{T} \tilde{q} \partial_{T}^{i j} p .
\end{aligned}
$$

Hence

$$
\int_{\tilde{T}} \tilde{q} \partial_{\tilde{T}}^{i j} \tilde{p}=\left\|B_{T}\right\|^{2} \int_{\tilde{T}} \widetilde{q} \widetilde{\partial_{T}^{i j}} p, \quad \forall \tilde{q} \in N_{i j} .
$$

By the fact that $\widetilde{\partial_{T}^{i j}} p \in N_{i j}$, we obtain

$$
\partial_{\tilde{T}}^{i j} \tilde{p}=\left\|B_{T}\right\|^{2} \widetilde{\partial_{T}^{i j}} p
$$

and it follows that

$$
\left|\partial_{\tilde{T}}^{i j} \tilde{p}\right|_{0, \tilde{T}}=\left\|B_{T}\right\|^{1 / 2}\left|\partial_{T}^{i j} p\right|_{0, T} .
$$

By (3.23), (3.24) and (3.25), we obtain (3.18).

Using the first two conclusions of the lemma and the interpolation theory, we can prove (3.19).

3.5. Remarks. In the coding for real computation, one prefers to use the degrees of freedom at the element vertices than ones at the relative interior of element edges and facets. In this sense, the incomplete cubic tetrahedral element, the three-dimensional Zienkiewicz element and the quasi-conforming tetrahedral element seem better than the cubic tetrahedral element. Although the interpolation error (or the approximation error) of the cubic tetrahedral element is one order higher than those of the incomplete cubic tetrahedral element and the quasiconforming element, the error of these elements, to solve the boundary value problem of fourth order partial differential equation, are all the same order (see the next section). As for the three-dimensional Zienkiewicz element, it is divergent and cannot be used.

Using a nonconforming element in real computation for problem (2.1), one needs to derive the second order derivatives of the shape functions and to write these 
derivatives in a form with respect to the vector of degrees of freedom. For the quasi-conforming tetrahedral element above, this has been given by (3.15), though the description of the element looks more complicated than ones of the cubic and incomplete cubic tetrahedral elements.

\section{Convergence analysis}

In this section, we discuss the convergence properties of the new elements in previous sections. Toward the end of this section, we show that the three-dimensional Zienkiewicz element is not convergent in general.

First, let us derive the error estimates for the interpolation operator.

Theorem 4.1. Let $\Pi_{T}$ be the interpolation operator corresponding to the cubic tetrahedral element, the incomplete cubic tetrahedral element, and the three-dimensional Zienkiewicz elements. Then there exists a constant $C$ independent of $h$ such that

$$
\left|v-\Pi_{T} v\right|_{m, T} \leq C h^{r-m}|v|_{r, T}, \quad 0 \leq m \leq r, \forall v \in H^{r}(T),
$$

where $r=4$ for the cubic tetrahedral element and $r=3$ for the other two elements.

From Lemma 3.1 and the argument 21 for the Morley element, we can show the following lemma.

Lemma 4.2. Let $V_{h 0}$ be the finite element space of the cubic tetrahedral element or the incomplete cubic tetrahedral element. Then there exists a constant $C$ independent of $h$ such that for $v \in H^{3}(\Omega) \cap H_{0}^{2}(\Omega)$ with $\Delta^{2} v \in L^{2}(\Omega)$,

$$
\left|a_{h}\left(v, v_{h}\right)-\left(\Delta^{2} v, v_{h}\right)\right| \leq C h\left(|v|_{3, \Omega}+h\left\|\Delta^{2} v\right\|_{0, \Omega}\right)\left|v_{h}\right|_{2, h}, \quad \forall v_{h} \in V_{h 0} .
$$

Now let $u$ and $u_{h}$ be the solutions of problems (2.4) and (2.6), respectively. Combining Theorem 4.1 and Lemma 4.2, we get the following theorem.

Theorem 4.3. Let $V_{h 0}$ be the finite element space of the cubic tetrahedral element or the incomplete cubic tetrahedral element. Then

$$
\lim _{h \rightarrow 0}\left\|u-u_{h}\right\|_{2, h}=0,
$$

and there exists a constant $C$ independent of $h$ such that

$$
\left\|u-u_{h}\right\|_{2, h} \leq C h\left(|u|_{3, \Omega}+h\|f\|_{0, \Omega}\right)
$$

when $u \in H^{3}(\Omega)$.

Proof. By the generalized Poincare-Friedrichs inequality (see [24]), we have

$$
\left\|v_{h}\right\|_{2, h}^{2} \leq C a_{h}\left(v_{h}, v_{h}\right), \quad \forall v_{h} \in V_{h 0}
$$

That is, $a_{h}(\cdot, \cdot)$ is uniformly $V_{h 0}$-elliptic. From the well-known Strang Lemma (see [8] or [22]),

$$
\left\|u-u_{h}\right\|_{2, h} \leq C\left(\inf _{w_{h} \in V_{h 0}}\left\|u-w_{h}\right\|_{2, h}+\sup _{w_{h} \in V_{h 0} w_{h} \neq 0} \frac{\left|a_{h}\left(u, w_{h}\right)-\left(f, w_{h}\right)\right|}{\left\|w_{h}\right\|_{2, h}}\right) .
$$

When $u \in H^{3}(\Omega)$, we obtain (4.4) directly from (4.5), (4.1), and (4.2).

For general case, we obtain from Lemma 3.1 and Green's formula that

$$
\sum_{T \in \mathcal{T}_{h}} \int_{T} \frac{\partial^{2} v_{h}}{\partial x_{i} \partial x_{j}}=0, \quad \forall v_{h} \in V_{h 0}, 1 \leq i, j \leq 3
$$


Then the patch test is passed on each $\mathcal{T}_{h}$. Because the assumption of Theorem 4.7 in [26] is satisfied, we obtain (4.3).

Now let $\Pi_{h}^{1}$ be the interpolation operator corresponding to the linear conforming element for the second order partial differential equation and $\mathcal{T}_{h}$. For the cubic tetrahedral element and the incomplete cubic tetrahedral elements, we can also consider another finite element method: to find $\tilde{u}_{h} \in V_{h 0}$ such that

$$
a_{h}\left(\tilde{u}_{h}, v_{h}\right)=\left(f, \Pi_{h}^{1} v_{h}\right), \quad \forall v_{h} \in V_{h 0} .
$$

For the finite element solution $\tilde{u}_{h}$ of problem (4.7), we can obtain its convergence and the error estimate.

Theorem 4.4. Let $V_{h 0}$ be the finite element space of the cubic tetrahedral element or the incomplete cubic tetrahedral element. Then

$$
\lim _{h \rightarrow 0}\left\|u-\tilde{u}_{h}\right\|_{2, h}=0,
$$

and there exists a constant $C$ independent of $h$ such that

$$
\left\|u-\tilde{u}_{h}\right\|_{2, h} \leq C h|u|_{3, \Omega}
$$

when $u \in H^{3}(\Omega)$.

For the convergence of the quasi-conforming tetrahedral element, we can follow the method used in [32] or 33]. We give the result without proof.

Theorem 4.5. For the quasi-conforming tetrahedral element, problem (3.17) has a unique solution $\bar{u}_{h}$ and

$$
\lim _{h \rightarrow 0}\left\|u-\bar{u}_{h}\right\|_{2, h}+\sum_{1 \leq i, j \leq 3}\left|\frac{\partial^{2} u}{\partial x_{i} \partial x_{j}}-\partial_{h}^{i j} \bar{u}_{h}\right|_{0, \Omega}=0
$$

and there exists a constant $C$ independent of $h$ such that

$$
\left\|u-\bar{u}_{h}\right\|_{2, h}+\sum_{1 \leq i, j \leq 3}\left|\frac{\partial^{2} u}{\partial x_{i} \partial x_{j}}-\partial_{h}^{i j} \bar{u}_{h}\right|_{0, \Omega} \leq C h|u|_{3, \Omega}
$$

when $u \in H^{3}(\Omega)$.

It is known that the Zienkiewicz element is not convergent for general meshes in two dimensions (see 20]). As an analogue in the three-dimensional case, the three-dimensional Zienkiewicz element has the same divergence property. Now we show that it is divergent for a special grid.

In Figure 3, a cube is divided into eight sub-cubes, and then each sub-cube is divided six tetrahedrons, where the tetrahedrons not represented by dashed lines are symmetric with respect to the centric point of the cube.

Now let $\Omega$ be the cube $[-1,1]^{3}$. For $k=1,2, \cdots$, let $\mathcal{T}_{k}$ be a triangulation of $\Omega$ defined as follows. First, $\Omega$ is subdivided into equal cubes with side length $h_{k}=2 / k$, and then each cube is subdivided into tetrahedrons such as the one shown in Figure 3 . The cases of $k=1$ and $k=2$ are shown in Figures 3 and 4 , respectively. 


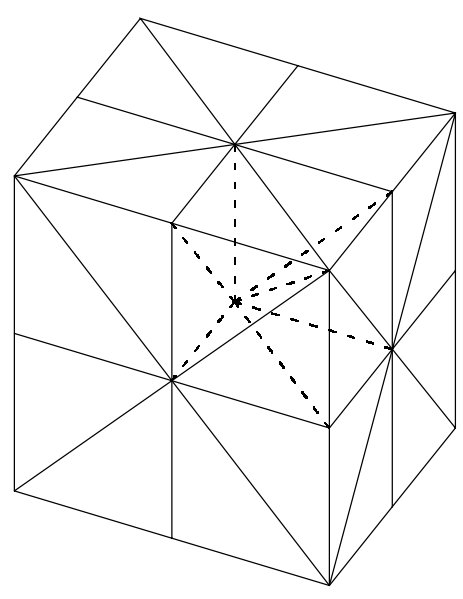

FiguRE 3

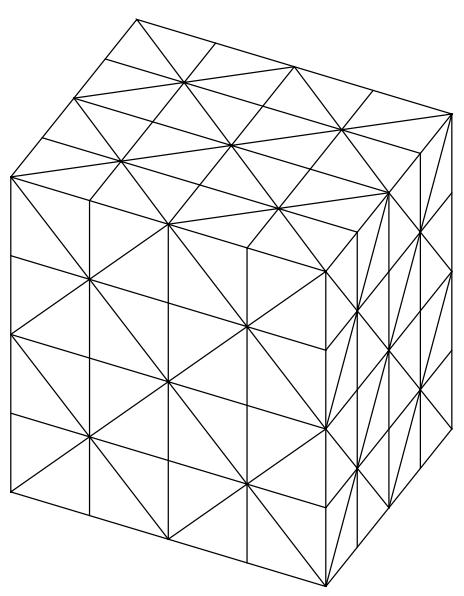

Figure 4

Theorem 4.6. The three-dimensional Zienkiewicz element is divergent for triangulations $\mathcal{T}_{k}$.

Proof. Let $V_{10}$ be the finite element space of the three-dimensional Zienkiewicz element on $\mathcal{T}_{1}$. Let $v_{h} \in V_{10}$ be the function such that $v_{h}$ is 1 at the center point of $\Omega$ and vanishes at other vertices of all elements in $\mathcal{T}_{1}$, and $D v_{h}$ vanishes at all vertices of all elements in $\mathcal{T}_{1}$. It can be computed that

$$
\begin{aligned}
& \sum_{T \in \mathcal{T}_{1}} \int_{T} \frac{\partial^{2} v_{h}}{\partial x_{i}^{2}}=-\frac{8}{3}, \quad i=1,2,3, \\
& \sum_{T \in \mathcal{T}_{1}} \int_{T} \frac{\partial^{2} v_{h}}{\partial x_{i} \partial x_{j}}=0, \quad 1 \leq i \leq 3, i<j \leq 3 .
\end{aligned}
$$

That is, the three-dimensional Zienkiewicz element does not pass the patch test. On the other hand, for $\mathcal{T}_{k}$ the number of the patches reduced from $\mathcal{T}_{1}$ is $k^{3}$, and the number of elements in $\mathcal{T}_{k}$ is $48 k^{3}$. By Theorem 6.1 in [26], we obtain the conclusion of the lemma.

\section{Concluding Remarks}

In this paper, we proposed and analyzed several tetrahedral complete or incomplete cubic finite elements for fourth order elliptic partial differential operators.

More work needs to be done for constructing other types of nonconforming elements. One noticeable element that is missing from our work is a three-dimensional extension of the Morley triangular element in two dimensions that only makes use of quadratic polynomials (although the cubic tetrahedral element, a $P_{3}$-element in three-dimensions, may be viewed as a $3 \mathrm{D}$ extension of the $P_{2}$-Morley element in 2D). As it turns out, in three and higher dimensions, the construction of the $P_{2}$-Morley element is possible, but it no longer uses the element vertices as part of the degrees of freedom. We will report an extension of the Morley element in any dimensions in [28]. Another type of element is the hexehedral nonconforming 
element that may be extended from rectangular nonconforming elements in two dimensions. We will report these extensions in any dimensions in [27.

\section{REFERENCES}

1. Alfeld P, A trivariate Clough-Tocher scheme for tetrahedral data, Comput. Aided Geom. Design, 1 (1984), 169-181.

2. Barrett J W and Blowey J F, Finite element approximation of the Cahn-Hilliard equation with concentration dependent mobility, Math. Comp., 68 (1999), 487-517. MR.1609678 (99i:65103)

3. Barrett J W, Blowey J F and Garcke H, Finite element approximation of the Cahn-Hilliard equation with degenerate mobility, SIAM J. Numer. Anal., 37, 1(1999), 286-318. MR 1742748 (2001c:65118)

4. Bazeley G P, Cheung Y K, Irons B M and Zienkiewicz O C, Triangular elements in plate bending - conforming and nonconforming solutions, in Proceedings of the Conference on Matrix Methods in Structural Mechanics, Wright Patterson A. F. Base, Ohio, 1965, 547-576.

5. Boettinger W J, Warren J A, Beckermann C and Karma A, Phase-field simulation of solidification, Annu. Rev. Mater. Res., 32, 1(2002), 63-94.

6. Cahn J W, Hilliard J E, Free energy of a nonuniform system I. Interfacial free energy, $J$. Chem. Phys., 28 (1958), 258-267.

7. Chen Wanji, Liu Yingxi and Tang Limin, The formulation of quasi-conforming elements, Journal of Dalian Institute of Technology, 19, 2(1980), 37-49.

8. Ciarlet P G, The Finite Element Method for Elliptic Problems, North-Holland, Amsterdam, New York, 1978. MR1930132

9. Du Qiang and Nicolaides R A, Numerical analysis of a continuum model for phase transition, SIAM J. Numer. Anal., 28 (1991), 1310-1322. MR.1119272 (92h:65166)

10. Elliott $\mathrm{C} \mathrm{M}$ and French D A, A nonconforming finite element method for the two dimensional Cahn-Hilliard equation, SIAM J. Numer. Anal., 26 (1989), 884-903. MR.1005515 (90k:65163)

11. Elliott $\mathrm{C} M$ and Larsson S, Error estimates with smooth and non-smooth data for a finite element method for the Cahn-Hilliard equation, Math. Comp., 58 (1992) 603-630, S33-S36. MR:1122067 (92f:65116)

12. Elliott C M, and Zhang S M, On the Cahn-Hilliard equation, Arch. Rational Mech. Anal., 96 (1986), 339-357.

13. Gatti S, et al., Hyperbolic relaxation of the viscous Cahn-Hilliard equation in $3-\mathrm{D}, M^{3} A S, \mathbf{1 5}$, 2(2005), 165-198. MR2119676 (2006c:35257)

14. Lai M J and LeMehaute A, A new kind of trivariate $C^{1}$ macro-element, Advances in Computational Mathematics, 21(2004), 273-292. MR2073143 (2005c:65103)

15. Landau L and Lifchitz E, Theory of Elasticity, Pergamon Press, London, 1959.

16. Lascaux $\mathrm{P}$ and Lesaint $\mathrm{P}$, Some nonconforming finite elements for the plate bending problem, RAIRO Anal. Numer., R-1 (1985), 9-53. MR0423968 (54:11941)

17. LeMehaute A, Interpolation et approximation par des fonctions polynomiales par morceaux dans $R^{n}$, Ph.D. Thesis, Univ. Rennes, France, 1984.

18. Morley L S D, The triangular equilibrium element in the solution of plate bending problems, Aero. Quart., 19 (1968), 149-169.

19. Seol D J, Hu S Y, Li Y L, Shen J, Oh K H and Chen L Q, Computer simulation of spinodal decomposition in constrained films, Acta Materialia, 51 (2003), 5173-5185.

20. Shi Zhong-ci, The generalized patch test for Zienkiewicz's triangles, J. Computational Mathematics, 2 (1984), 279-286. MR0815422 (86m:73051)

21. Shi Zhong-ci, On the error estimates of Morley element, Numerica Mathematica Sinica 12, 2(1990), 113-118.

22. Strang G and Fix G J, An Analysis of the Finite Element Method, Prentice-Hall, Englewood Cliffs, 1973. MR0443377 (56:1747)

23. Stummel F, The generalized patch test, SIAM J. Numer. Analysis, 16 (1979), 449-471. MR.0530481 (80e:65106)

24. Stummel F, Basic compactness properties of nonconforming and hybrid finite element spaces, RAIRO, Anal. Numer., 4, 1(1980), 81-115. MR0566091(81h:65058)

25. Tang Limin, Chen Wanji and Liu Yingxi, Quasi-conforming elements in finite element analysis, J. Dalian Inst. of Technology, 19, 2(1980), 19-35. 
26. Wang Ming, On the necessity and sufficiency of the patch test for convergence of nonconforming finite elements, SIAM J. Numer. Anal., 39, 2(2002), 363-384. MR.1860273 (2002h:65197)

27. Wang Ming, Shi Zhong-ci and Jinchao Xu, Some $n$-rectangle nonconforming elements for fourth order elliptic equations, Research Report, 50(2005), School of Mathematical Sciences and Institute of Mathematics, Peking University; J. Comput. Math., to appear.

28. Wang Ming and Jinchao Xu, The Morley element for fourth order elliptic equations in any dimensions, Research Report, 89 (2004), School of Mathematical Sciences and Institute of Mathematics, Peking University. Numer. Math., 103 (2006), 155-169.

29. Worsey A J and Farin G, An n-dimensional Clough-Tocher interpolant, Constr. Approx., 3 (1987), 99-110. MR0889547 (89f:41007)

30. Zenicek A, Polynomial approximation on tetrahedrons in the finite element method, J. Approx. Theory, 7 (1973), 334-351.

31. Zenicek A, A general theorem on triangular finite $C^{(m)}$-elements, RAIRO Anal. Numer., R-2 (1974), 119-127.

32. Zhang Hongqing and Wang Ming, On the compactness of quasi-conforming element spaces and the convergence of quasi-conforming element method, Appl. Math. Mech. (English edition), 7 (1986), 443-459.

33. Zhang Hongqing and Wang Ming, The Mathematical Theory of Finite Elements, Science Press, Beijing, 1991.

Lmam, School of Mathematical Sciences, Peking University, Beijing, People's RePUBLIC OF CHINA

E-mail address: mwang@math.pku.edu.cn

The School of Mathematical Sciences, Peking University; Beijing, People's Republic of China; and Department of Mathematics, Pennsylvania State University, University Park, Pennsylvania 16802

E-mail address: xu@math.psu.edu 